\title{
Wavelength Assignment for Parallel FFT Communication Pattern on Linear Arrays by Lattice Embedding*
}

\author{
Yawen Chen, Hong Shen \\ Japan Advanced Institute of Science and Technology \\ Asahidai 1-8, Nomi-Shi, Ishikawa, Japan, 923-1292 \\ Email:\{yawen, shen\} @jaist.ac.jp
}

\begin{abstract}
Fast Fourier Transform(FFT) represents a common communication pattern shared by a large class of scientific and engineering problems and wavelength assignment is a key issue to increase efficiency and reduce cost in Wavelength Division Multiplexing (WDM) optical networks. In this paper, we propose a new scheme for the wavelength assignment of parallel FFT communication pattern on WDM linear arrays. By lattice embedding, the number of wavelengths required to realize parallel FFT communication pattern on WDM linear arrays significantly improves the known result. Our proposed embedding method also provides a new approach to the hypercube layout problem considering connections dimension by dimension rather than all connections as in the traditional approach.
\end{abstract}

\section{Introduction}

Fast Fourier Transform (FFT) plays an important role in numerous scientific and technical applications. While the application fields of FFT are growing rapidly, the amount of data to be transformed is also increasing tremendously. Hence, there has been a great interest in implementing FFT on parallel computers and some parallel computers have been specially designed to perform FFT computations [2]. With the increasing computation power of parallel computers, interprocessor communication has become an important factor that limits the performance of supercomputing systems. Optical communication, in particular, Wavelength Division Multiplexing (WDM) technique, has become a promising technology for many emerging networking and parallel/distributed computing applications because of its huge bandwidth [7].

As we know, parallel FFT is often implemented on dense interconnection networks such as hypercube and shuffleexchange networks due to its topological properties [2].
Since WDM divides the bandwidth of an optical fiber into multiple wavelength channels so that multiple devices can transmit on distinct wavelengths through the same fiber concurrently, these dense networks can be simplified to simple regular topologies by realizing connections of parallel FFT communication patterns in optical lightpaths.

To efficiently utilize the bandwidth resources and to eliminate the high cost and bottleneck caused by optoelectronic conversion and processing at intermediate nodes, end-to-end lightpaths are usually set up between each pair of source-destination nodes. A connection or a lightpath in a WDM network is an ordered pair of nodes $(x, y)$ corresponding to that a packet is sent from source $x$ to destination $y$. In this paper, we assume that no wavelength converter facility is available in the network. Thus, a connection must use the same wavelength throughout its path. In this case, the lightpath is said to satisfy the wavelengthcontinuity constraint.

RWA tries to minimize the number of channels to realize a communication requirement by taking into consideration both routing options and channel assignment options which can be described as follows [6]: Given a set of alloptical connections, the problem is to (a) find routes from the source nodes to their respective destinations, and (b) assign channels to these routes so that the same channel is assigned to all the links of a particular route. (c) The goal of RWA is to minimize the number of assigned channels. Numerous research studies have been conducted on the RWA problem. A review of routing and wavelength assignment approaches for wavelength-routed optical WDM networks was given in [8]. In [6], optimal routing and channel assignments for hypercube communication on optical meshlike processor arrays were studied. In [5], multicasting in multi-hop optical WDM networks with limited wavelength conversion was surveyed. In [4], routing and wavelength assignment for parallel LU decomposition communication pattern on WDM ring was studied.

In [3], the problem of wavelength assignment for realizing parallel FFT communication pattern on a class of regu- 
lar optical WDM networks was addressed and two methods, sequential mapping and shift-reversal mapping, were proposed. In this paper, we design a new scheme for the wavelength assignment of parallel FFT communication pattern on WDM linear arrays. By lattice embedding, the number of wavelengths required to realize parallel FFT communication pattern on WDM linear arrays significantly improves the known result in [3]. Our results have a clear significance for applications because FFT represents a common communication pattern shared by a large class of scientific and engineering problems and WDM optical networks as a promising technology in networking has an increasing popularity. Our proposed embedding method also provides a new approach to the hypercube layout problem considering connections dimension by dimension rather than all connections as in the traditional approach.

The rest of this paper is organized as follows. In Section 2, we define the problem of wavelength assignment for parallel FFT communication pattern on WDM linear arrays. We then design lattice embedding and provide some results of wavelength assignment for parallel FFT communication pattern on linear arrays in Sections 3. Finally, we conclude this paper in Section 4.

\section{Problem Definition}

FFT developed by Cooley and Tukey in the mid-60s is a method of computing the discrete Fourier transform which reduces the number of operations for an $N$-point complex vector from $O\left(N^{2}\right)$ to $O\left(N \log _{2} N\right)$. The data-flow graph induced by an $N$-point FFT computation is usually described by means of the so-called butterfly representation [2]. The butterfly representation of FFT algorithm is a diagram made up of blocks representing identical computational units (butterflies) connected by arrows that show the flow of data between the blocks. Assuming that $N$ is the length of the sequence to be transformed ( $N$ is an integer power of two), then the diagram with $N\left(\log _{2} N+1\right)$ nodes arranged in $N$ rows and $\log _{2} N+1$ columns is made of $\log _{2} N$ stages of $N / 2$ butterflies each.

Generally, FFT is implemented stage by stage, i.e. any stage of calculation cannot proceed until all the results of its previous stage have been completed. In this paper, we consider one dimensional data sequence of size $N=2^{n}$. If each data is assigned a binary representation, the communications during the $i$ th $(1 \leq i \leq n)$ stage of the butterfly must take place between the nodes whose binary representations differ in the $i$ th bit. If the butterfly representation is viewed as a process graph, i.e. each row of the butterfly is implemented by a process and each arrow by a communication channel, it is apparent that the butterfly communication pattern can map onto a WDM hypercube perfectly those links connecting the nodes having an address that differs by only one bit at each stage. However, if a WDM hypercube is used, only the $i$ th dimensional links are used with one wavelength during the $i$ th stage whereas other $(n-1) \times 2^{n-1}$ links are vacant during this stage, which may lead to wasting of wavelength channels. As we know, a connection in the hypercube communication pattern is called a dimensional $i$ connection [6] if it connects two nodes that differ in the $i$ th bit position, where $1 \leq i \leq n$. In a network of size $2^{n}$, the set $D I M_{i}$ is defined as the set of all dimension $i$ connections and $H_{n}$ is defined as the hypercube communication pattern which contains all connections in the hypercube. That is, $H_{n}=\bigcup_{i=1}^{n} D I M_{i}$ and $D I M_{i}=\left\{\left(j, j+(-1)^{\left\lfloor j / 2^{n-i}\right\rfloor} \times 2^{n-i}\right) \mid 0 \leq j \leq 2^{n}-1\right\}$. With input data distributed on processors, the set of all communications during $n$ stages of parallel FFT is equivalent to $H_{n}$, and the set of communications during the $i$ th stage is equivalent to $D I M_{i}$. Clearly, parallel FFT has a regular communication pattern which we denote by $F F T_{n}(n \geq 2)$.

We model a network as a directed graph $G(V, E)$. Nodes in $V$ are switches and edges in $E$ are links. In general, an optical WDM network consists of routing nodes interconnected by point-to-point fiber links, which can support a certain number of wavelengths. In this paper, we assume each link in the network is bidirectional and composed of a pair of unidirectional links with one link in each direction. For $F F T_{n}$, if $(x, y) \in F F T_{n}$, then $(y, x) \in F F T_{n}$. Assuming that these two communications can be realized by two lightpaths in the same path of opposite directions passing through different fiber links, the same wavelength can be assigned to these two lightpaths. In this case, we can ignore the problem of communication directions in $F F T_{n}$.

It should be noted that $F F T_{n} \neq H_{n}$ because the number of wavelengths required to realize $F F T_{n}$ on the WDM optical networks is not equal to that required to realize hypercube communications of $H_{n}$. Since the $n$ stages of parallel FFT should be implemented stage by stage, the number of wavelengths required to realize $F F T_{n}$ on optical WDM networks is the maximum number among the wavelengths required by the $n$ stages. Let $W_{e}(P$, line $)$ denote the number of wavelengths required to realize communication pattern $P$ on linear arrays by embedding scheme $e$. Thus, $W_{e}\left(F F T_{n}\right.$, line $)=\max _{1 \leq i \leq n}\left(W_{e}\left(D I M_{i}\right.\right.$, line $\left.)\right)$.

The problem of wavelength assignment for $F F T_{n}$ on WDM linear arrays can be regarded as the problem of embedding the hypercube communications on linear arrays, such that the maximum congestion of dimensional $i$ connections, for $1 \leq i \leq n$, is minimized. This problem arising from the wavelength assignment of $F F T_{n}$, which focuses on minimizing the congestion of each dimensional connections, is different from the traditional embedding of hypercube on linear arrays to minimize congestion which considers all the connections on hypercube. 


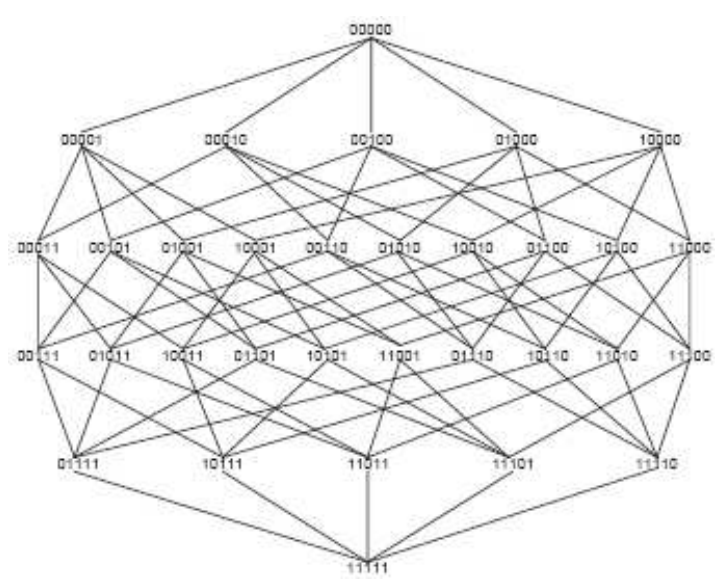

Figure 1. $H_{5}$ represented as a lattice

\section{Lattice Embedding of Parallel FFT on Lin- ear Arrays}

\subsection{Lattice Embedding}

We design a new embedding scheme on linear arrays, lattice embedding, which is based on the lattice form of hypercube. Firstly, we introduce the following properties of hypercube in a form of lattice.

Hypercube can be represented in a form of lattice, as shown in Figure 1. As we know, for the hypercube with $2^{n}$ nodes, each node corresponds to an $n$-bit binary representation, and two nodes are linked with a connection if and only if their binary representations differ in precisely one bit. For the lattice form of $2^{n}$-node hypercube, there are $n+1$ node rows connected by the hypercube connections. If the rows are numbered from top to bottom in ascending order starting from 0 , the binary representations of the nodes on the $k$ th row, for $0 \leq k \leq n$, have $k 1$ s and $n-k 0$ s. In addition, hypercube connections only exist between the nodes on two neighborhood rows. Such a lattice form of hypercube also has the following properties:

Property 1: Hypercube with $2^{n}$ nodes can be represented in a form of lattice with $\left(\begin{array}{l}n \\ k\end{array}\right)$ nodes on the $k$ th row for $0 \leq k \leq n$.

Proof: As the number of nodes whose binary representations have $k 1$ s among $n$ bits is $\left(\begin{array}{l}n \\ k\end{array}\right)$, it is easy to know the number of nodes on the $k$ th row of the hypercube lattice is $\left(\begin{array}{l}n \\ k\end{array}\right)$.

Property 2: Hypercube with $2^{n}$ nodes can be represented in a form of lattice with $n \times\left(\begin{array}{c}n-1 \\ k\end{array}\right)$ connections connecting the nodes of the $k$ th row and the $(k+1)$ th row, for $0 \leq k \leq n-1$.

Proof: Since the number of $1 \mathrm{~s}$ on the $k$ th row and $(k+1)$ th row are $k$ and $k+1$ respectively, node $u$ on the $k$ th row connects with those nodes on the $(k+1)$ th row whose binary representations have $k 1$ s on the same positions with $u$. As the number of nodes on the $(k+1)$ th row with $k 1$ s on the same positions with $u$ is $n-k$, each node on the $k$ th row has $n-k$ connections with the nodes on the $(k+1)$ th row. Therefore, the number of connections between the nodes of the $k$ th row and $(k+1)$ th row is $(n-k) \times\left(\begin{array}{l}n \\ k\end{array}\right)=n \times\left(\begin{array}{c}n-1 \\ k\end{array}\right)$.

Property 3: Hypercube with $2^{n}$ nodes can be represented in a form of lattice with $\left(\begin{array}{c}n-1 \\ k\end{array}\right)$ dimensional $i$ connections, for $1 \leq i \leq n$, connecting the nodes of the $k$ th row and the $(k+1)$ th row for $0 \leq k \leq n-1$.

Proof: If node $u$ is on the $k$ th row whose $i$ th bit is 0 , then $u$ must connect with one of the nodes on the $(k+1)$ th row by dimensional $i$ connection. It is easy to know the number of nodes on the $k$ th row, whose binary representations have $k 1$ s and $i$ th bit is 0 , is $\left(\begin{array}{c}n-1 \\ k\end{array}\right)$. Therefore, the number of dimensional $i$ connections, for $1 \leq i \leq n$, connecting the nodes of the $k$ th row and the $(k+1)$ th row is $\left(\begin{array}{c}n-1 \\ k\end{array}\right)$.

It can be seen from the above properties that the number of dimensional $i$ connections is identical with each $1 \leq i \leq$ $n$.

Assume that the nodes of WDM linear arrays are numbered from left to right in ascending order starting from 0 , and that the links are numbered from left to right starting from 1. Define each node numbering of $H_{n}$ by a function $\eta: V_{H_{n}} \mapsto\left\{0,1, \ldots, 2^{n}-1\right\}$ which is a one-to-one mapping from the nodes of $H_{n}$ to the nodes of an $2^{n}$-node linear array. Let $R(u)=k$ if node $u$ is on the $k$ th row of hypercube lattice. Embed the nodes of the $2^{n}$-node hypercube lattice from row 0 to row $n$ onto the $2^{n}$-node linear array from left to right node by node. That is to say, if $R(u)<R(v)$, then $\eta(u)<\eta(v)$. We call the above embedding way lattice embedding. Let $e^{n}(k)=\sum_{i=0}^{k}\left(\begin{array}{c}n \\ i\end{array}\right)=$ $\left(\begin{array}{l}n \\ 0\end{array}\right)+\left(\begin{array}{l}n \\ 1\end{array}\right)+\left(\begin{array}{l}n \\ 2\end{array}\right)+\ldots+\left(\begin{array}{l}n \\ k\end{array}\right)$. By the definition of lattice embedding, the node on the row 0 of hypercube lattice is mapped onto node 0 of the linear array, and nodes on the $k$ th row of $2^{n}$-node hypercube lattice, for $1 \leq k \leq n$, are mapped between node $e^{n}(k-1)$ and node $e^{n}(k)-1$ on the $2^{n}$-node linear array. That is to say, if $R(u)=k$, then $e^{n}(k-1) \leq \eta(u) \leq e^{n}(k)-1$.

\subsection{Wavelength Assignment of Parallel FFT on Linear Arrays}

Let $\omega_{l}^{i j}$ be the number of wavelengths required by lattice embedding to realize $F F T_{n}$ on the $j$ th link of the linear arrays during the $i$ th stage and $\omega_{l}^{i}$ be the maximum number of wavelengths required among all the links of the linear arrays during the $i$ th stage. Therefore, the number of wavelengths required to realize $F F T_{n}$ by lattice embedding, denoted by $\omega_{l}$, is

$$
\omega_{l}=\max _{1 \leq i \leq n} \omega_{l}^{i}=\max _{1 \leq i \leq n}\left(\max _{1 \leq j \leq 2^{n-1}} \omega_{l}^{i j}\right)
$$


By lattice embedding, the following results can be obtained.

Theorem 1: By lattice embedding, the number of wavelengths required to realize $F F T_{n}$ is not less than $\left(\begin{array}{c}n-1 \\ \lfloor(n-1) / 2\rfloor\end{array}\right)$.

Proof: By Property 2 and 3, all the hypercube connections are only between the nodes of the $k$ th row and the $(k+1)$ th row with $\left(\begin{array}{c}n-1 \\ k\end{array}\right)$ dimensional $i$ connections, for $0 \leq k \leq n-1$ and $1 \leq i \leq n$. So, the number of dimensional $i$ connections passing through link $e^{n}(k)$ are $\left(\begin{array}{c}n-1 \\ k\end{array}\right)$. Therefore, the number of wavelengths required to realize $F F T_{n}$ by lattice embedding satisfies, $\omega_{l}=\max _{1 \leq i \leq n} \omega_{l}^{i}$ $=\max _{1 \leq i \leq n}\left(\max _{1 \leq j \leq 2^{n-1}} \omega_{l}^{i j}\right) \geq \max _{0 \leq k \leq n-1}\left(\begin{array}{c}n-1 \\ k\end{array}\right)=$ $\left(\begin{array}{c}n-1 \\ \lfloor(n-1) / 2\rfloor\end{array}\right)$.

Theorem 2: By lattice embedding, the number of wavelengths required to realize $F F T_{n}$ is not more than $\left(\begin{array}{c}n \\ \lfloor n / 2\rfloor\end{array}\right)$

Proof: On the $k$ th row of the hypercube lattice for $1 \leq k \leq n-1$, there are $\left(\begin{array}{l}n-1 \\ k-1\end{array}\right)$ nodes, whose $i$ th bit is 1 , connecting the nodes on the $(k-1)$ th row by dimensional $i$ connections and $\left(\begin{array}{c}n-1 \\ k\end{array}\right)$ nodes, whose $i$ th bit is 0 , connecting the nodes on the $(k+1)$ th row by dimension $i$ connections. Therefore, the number of dimensional $i$ connections passing thought the links from $e^{n}(k-1)+1$ to $e^{n}(k)-1$ is not more than the sum of the maximum number of dimensional $i$ connections between the $k$ th row with its two neighborhood rows. Therefore, the number of wavelengths required to realize $F F T_{n}$ satisfies, $\omega_{l}=$ $\max _{1 \leq i \leq n}\left(\max _{1 \leq j \leq 2^{n-1}} \omega_{l}^{i j}\right) \leq \max _{1 \leq k \leq n-1}\left(\left(\begin{array}{c}n-1 \\ k\end{array}\right)+\right.$ $\left.\left(\begin{array}{l}n-1 \\ k-1\end{array}\right)\right)=\max _{1 \leq k \leq n-1}\left(\begin{array}{l}n \\ k\end{array}\right)=\left(\begin{array}{c}n \\ \lfloor n / 2\rfloor\end{array}\right)$

As $n ! \sim \sqrt{2 \pi n}(n / e)^{n}$ for large $n$ according to Stirling's Formula, it can be calculated from Theorem 1 and 2 that realizing $F F T_{n}$ requires $O\left(2^{n} / \sqrt{n}\right)$ wavelengths for large $n$. It can be concluded that lattice embedding outperforms the known embedding approaches in [3]. Theorem 1 and Theorem 2 give a rough estimation of the number of wavelengths required to realize $F F T_{n}$ on linear arrays. Furthermore, we discuss a computation method for the lattice embedding.

Theorem 3: The minimum number of wavelengths required to realize $F F T_{n}$ on $2^{n}$-node linear array by lattice embedding is $\left(\begin{array}{c}n-1 \\ \lfloor(n-1) / 2\rfloor\end{array}\right)+1$.

Proof: If $e^{n}(k-1) \leq j \leq e^{n}(k)-1$ for some $1 \leq k \leq$ $n-1$, let $\omega_{l 1}^{i j}$ be the number of dimensional $i$ connections between nodes in $U=\left\{u \mid e^{n}(k-1) \leq \eta(u) \leq j-1\right\}$ and nodes in $U^{\prime}=\left\{u^{\prime} \mid R\left(u^{\prime}\right)=k+1\right\}$, and $\omega_{l 2}^{i j}$ be the number of dimensional $i$ connections between nodes in $V=$ $\left\{v \mid j \leq \eta(v) \leq e^{n}(k)-1\right\}$ and nodes in $V^{\prime}=\left\{v^{\prime} \mid R\left(v^{\prime}\right)=\right.$ $k-1\}$. As the connections only take place between the neighborhood rows, the number of dimension $i$ connections passing through the $j$ th link on the linear array is $\omega_{l 1}^{i j}+\omega_{l 2}^{i j}$. Define $\eta^{-1}(j)$ as the node of $F F T_{n}$ which is mapped onto the $j$ th node of the linear array. Let $\theta_{i}(u)=1$ if the $i$ th bit of $u$ is 1 and $\theta_{i}(\neg u)=1$ if the $i$ th bit of $u$ is 0 . From the properties of hypercube lattice, it is easy to know that $u$ connects with a node in $V^{\prime}=\left\{v^{\prime} \mid R\left(v^{\prime}\right)=k-1\right\}$ by dimensional $i$ connection if $\theta_{i}(u)=1$, or with a node in $U^{\prime}=\left\{u^{\prime} \mid R\left(u^{\prime}\right)=k+1\right\}$ by dimensional $i$ connection if $\theta_{i}(\neg u)=1$. Therefore, $\omega_{l 1}^{i j}=\sum_{u=\eta^{-1}\left(e^{n}(k-1)\right)}^{\eta^{-1}(j-1)} \theta_{i}(\neg u)$ and $\omega_{l 2}^{i j}=\sum_{u=\eta^{-1}(j)}^{\eta^{-1}\left(e^{n}(k)-1\right)} \theta_{i}(u)$.

As the number of nodes whose $i$ th bit is 0 in $U \bigcup V$ is $\left(\begin{array}{c}n-1 \\ k\end{array}\right)$, and that in $U$ is $\omega_{l 1}^{i j}$, the number of nodes in $V$ whose $i$ th bit is 1 can also be calculated by $\omega_{l 2}^{i j}=\left(e^{n}(k)-j\right)-$ $\left(\left(\begin{array}{c}n-1 \\ k\end{array}\right)-\omega_{l 1}^{i j}\right)=e^{n}(k)-\left(\begin{array}{c}n-1 \\ k\end{array}\right)+\omega_{l 1}^{i j}-j$.

Therefore, the number of wavelengths required to realize $F F T_{n}$ on linear arrays can be calculated by the following equation: $\omega_{l}=$ $\max _{1 \leq i \leq n, 1 \leq k \leq n-1}\left(\max _{e^{n}(k-1) \leq j \leq e^{n}(k)-1}\left(\omega_{l 1}^{i j}+\omega_{l 2}^{i j}\right)\right)=$ $\max _{1 \leq i \leq n, 1 \leq k \leq n-1}\left(\max _{e^{n}(k-1) \leq j \leq e^{n}(k)-1}\left(2 \omega_{l 1}^{i j} \quad+\right.\right.$ $\left.\begin{array}{lllll}e^{n}(k) & - & \left(\begin{array}{c}n-1 \\ k\end{array}\right) \quad- & j\end{array}\right)$ = $\max _{1 \leq i \leq n, 1 \leq k \leq n-1}\left(\max _{e^{n}(k-1) \leq j \leq e^{n}(k)-1}\left(2 \omega_{l 1}^{i j} \quad+\right.\right.$ $\left.\left.e^{n}(k)-\left(\begin{array}{c}n-1 \\ k\end{array}\right)-j\right)\right)$.

For $n$ is even, $\omega_{l}=\max \left\{2 \sum_{u=\eta^{-1}\left(e^{n}(n / 2-1)\right)}^{\eta^{-1}(j-1)} \theta_{i}(\neg u)+\right.$ $e^{n}(n / 2)-\left(\begin{array}{c}n-1 \\ n / 2\end{array}\right)-j: 1 \leq i \leq$ $\left.n, e^{n}(n / 2-1) \leq j \leq e^{n}(n / 2)-1\right\}$. Since each node has $\mathrm{n} / 2 \quad 0 \mathrm{~s}$ on the $(n / 2)$ th row, $\sum_{i=1}^{n} \sum_{u=\eta^{-1}\left(e^{n}(n / 2-1)\right)}^{\eta^{-1}(j-1)} \theta_{i}(\neg u)=\frac{n}{2} \times\left(j-e^{n}(n / 2-1)\right)$. So, $\quad \max _{1 \leq i \leq n}\left(\sum_{u=\eta^{-1}\left(e^{n}(n / 2-1)\right)}^{\eta^{-1}(j-1)} \theta_{i}(\neg u)\right) \quad \geq$ $\left\lceil\frac{\frac{n}{2} \times\left(j-e^{n}(n / 2-1)\right)}{n}\right\rceil$. By the above equations, it can be calculated that $\omega_{l} \geq \max \left\{2 \times\left\lceil\frac{\frac{n}{2} \times\left(j-e^{n}(n / 2-1)\right)}{n}\right\rceil+e^{n}(n / 2)-\right.$ $\left.\left(\begin{array}{c}n-1 \\ n / 2\end{array}\right)-j, e^{n}(n / 2-1) \leq j \leq e^{n}(n / 2)-1\right\}=\left(\begin{array}{c}n-1 \\ n / 2-1\end{array}\right)+1$. Similarly, it can be calculated that for $n$ is odd, $\omega_{l} \geq$ $\left(\begin{array}{c}n-1 \\ (n-1) / 2\end{array}\right)+1$

From the proving of Theorem 3, it can be observed that the minimum number of wavelengths required by lattice embedding can be achieved if the 0 s are distributed as much as evenly among the $n$ bits of the number of 0 s on the nodes before each node $j$ for $e^{n}(n / 2-1) \leq j \leq e^{n}(n / 2)-1$.

For $n$ is even, the number of 0 s of the nodes in $U=$ $\left\{u \mid e^{n}(n / 2-1) \leq \eta(u) \leq e^{n}(n / 2)-1\right\}$ is $n / 2$. If $u$ is in $U, \neg u$ must be in $U$. So, $U=\left\{u_{i} \bigvee \neg u_{i} \mid 1 \leq\right.$ $\left.i \leq\left(\begin{array}{c}n \\ n / 2\end{array}\right) / 2\right\}$. Thus, there are $\left(\begin{array}{c}n \\ n / 2\end{array}\right) / 2$ such node pairs in $U$. The minimum number of wavelengths required by lattice embedding can be archived by mapping the nodes in $U$ on the $2^{n}$-node linear array pair by pair. That is to say, the number of wavelengths required is $\left(\begin{array}{c}n-1 \\ n / 2-1\end{array}\right)+1$ if we map $u_{1}, \neg u_{1}, u_{2}, \neg u_{2}, \ldots, u_{\left(\begin{array}{c}n \\ n / 2\end{array}\right) / 2}, \neg u_{\left(\begin{array}{c}n \\ n / 2\end{array}\right) / 2}$ onto the node from $e^{n}(n / 2-1)$ to $e^{n}(n / 2)-1$ respectively. For example, the number of wavelengths required to realize $F F T_{4}$ on 16-node linear array is 4 , if the nodes 0011,1100 , 0101, 1010, 1001, 0110 on $F F T_{4}$ are mapped onto the 


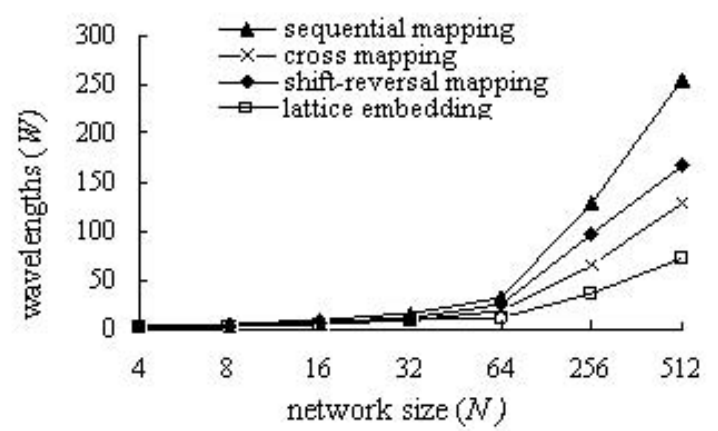

Figure 2. Comparisons on the number of wavelengths

nodes of 5,6,7,8,9,10 on the 16-node linear array respectively. The minimum number by lattice embedding can be discussed similarly for $n$ is odd. For example, if $n=5$, the nodes of $F F T_{5}$ which are mapped onto the nodes of $6,7,8,9,10,11,12,13,14,15$ on the 32 -node linear array can be 00011, 01100, 10001, 00110, 11000, 00101, 01010, 10100, 01001, 10010 respectively.

It can be seen that lattice mapping outperforms the known embedding approaches to realize FFT communication pattern on linear arrays on the number of wavelengths, as shown in Figure 2.

\section{Concluding Remarks}

In this paper, we propose a new scheme for the wavelength assignment of parallel FFT communication pattern on WDM linear arrays. By lattice embedding, the number of wavelengths required to realize parallel FFT communication pattern on WDM linear arrays significantly improves the known result in [3]. Our proposed embedding method also provides a new approach to the hypercube layout problem considering connections dimension by dimension rather than all connections as in the traditional approach. Our results have a clear significance for applications because FFT represents a common communication pattern shared by a large class of scientific and engineering problems and WDM optical networks as a promising technology in networking has an increasing popularity.

Since there are different communication patterns according to different parallel algorithms, how to realize these communication patterns on optical networks is a hot research field. Future work may include other type of optical networks and other RWA problems. More researches on RWA considering various parallel communication patterns for parallel algorithms may be a worthwhile effort.

Another interesting issue is to find the number of wave- lengths required on other types of WDM optical networks such as ring, mesh and torus based on the result in this paper. An open problem is to find the lower bounds for this problem and the improving schemes which can achieve the lower bounds.

\section{References}

[1] A. Averbuch, E. Ozdaglar and Dimitri P. Bertsekas. Routing and wavelength assignment in optical networks. IEEE/ACM Transactions on Networking, 11(2): 259-272, 2003.

[2] F. T. Leighton. Introduction to Parallel Algorithms and Architectures: Arrays, Trees, Hypercubes. Morgan Kaufmann Publishers, Inc., 1992.

[3] Fangai Liu and Yawen Chen. Wavelength Assignment of Parallel FFT Communication Pattern in a Class of Regular Optical WDM Network. Proceedings of the IEEE International Symposium on Parallel Architectures, Algorithms, and Networks, pp.495-500. IEEE Computer Society. Hong Kong, 2004.

[4] Yawen Chen and Fangai Liu. A Wavelength Assignment Algorithm of Parallel LU Decomposition Communication Pattern On WDM Ring Interconnection Network. International Symposium on Distributed Computing and Applications to Business, Engineering and Science, pp. 366-370. Wuhan, China, 2004.

[5] H. Shen, Y. Pan, J. Sum and S. Horiguchi, Multicasting in multihop optical WDM networks with limited wavelength conversion. IEICE Transactions on Information and Systems, E86-D(1):3-14, 2003.

[6] Yuan X and Melhcm R. Optimal Routing and Channel Assignments for Hypercube Communication on Optical Mesh-like Processor Arrays. Proceedings of the 5th International Conference on Massively Parallel Processing Using Optical Interconnection, pp.110-118. IEEE Computer Society Press. Las Vegas, NV, 1998.

[7] Yuanyuan Yang and Jianchao Wang. Cost-Effective Designs of WDM Optical Interconnects. IEEE Transactions on Parallel and Distributed Systems, 16(1): 5166, 2005.

[8] Hui Zang, Jason P. Jue, and Biswanath Mukherjee. A review of routing and wavelength assignment approaches for wavelength-routed optical WDM networks. SPIE Optical Networks Magazine, 1(1):47-60, 2000 . 